NEWS

\title{
Autism by the numbers: Explaining its apparent rise
}

\section{BY SPECTRUM}

22 DECEMBER 2021

Among autism's many mysteries is its apparent rise in prevalence around the world. But how common is it, exactly? And is it really more common among children today than in generations past? This new downloadable book from Spectrum offers an in-depth guide to the various factors - perhaps chief among them, a greater awareness of the condition and researchers' shifting ways of defining and measuring it - that have helped to drive autism prevalence numbers up.

Cite this article: https://doi.org/10.53053/WSYH3045 